\title{
Editorial: 2014 in Review
}

\author{
Fred R. Volkmar
}

Published online: 12 February 2015

(C) Springer Science+Business Media New York 2015

For the last several years, the number of papers submitted to the Journal has continued to increase steadily, from 740 submissions in 2012, to 822 in 2013, and 940 last year. Of these, less than a third (about $30 \%$ ) were ultimately accepted. Having expanded our editorial board and the number of associate editors, $J A D D$ continues to produce the highest quality articles despite the increased volume. In addition, the Journal has maintained an efficient turnaround time for the majority of reviews, averaging about 40 days before authors receive a first decision. Given the increased volume of papers the rejection rate, not surprisingly, has also risen. To conserve valuable Journal space, authors are encouraged to use online resources to publish extra supplementary, but still valuable, materials that can reasonably be included in online appendices.

As always, I'm deeply grateful to the Associate Editors: Joshua Diehl, Sandra Harris, Christopher McDougle, Ann S. Le Couteur, James McPartland, Nancy Minshew, Jeremy M. Veenstra-Vanderweele. Each has generously and diligently supported the work of the Journal, even in the face of the growing number of submissions. During the past year, we have also welcomed the following new Associate Editors Marc Woodbury-Smith, McMaster University, Carla Mazefsky, University of Pittsburgh Medical Center, and Susan White, Virginia Tech. We also acknowledge the hard work of our book editor, Brian Reichow, $\mathrm{PhD}$, at the University of Florida, who has also become an Associate Editor. In the Journal office, Lori Klein, Editorial Manager, and Isaac Smith, Editorial Assistant, have worked diligently to ensure that papers conform to editorial standards even before they enter the peer review process. We

F. R. Volkmar ( $\square)$

New Haven, CT, USA

e-mail: fred.volkmar@yale.edu also thank Judy Jones at Springer and the individuals involved in the production of the Journal including Garth Haller, Michelle Tam, Suganya Manikandan, and Bhanuprakash Sherla.

We welcome the following new members to the editorial board, all very worthy having submitted several reviews this past year: Jan Blacher, Graduate School of Education, University of California, Riverside, Nirit BaumingerZviely, Bar-Ilan University, Ramat-Gan, Israel, Gagan Joshi, Massachusetts General Hospital, Boston, MA, Oren Shtayermman, New York Institute of Technology, Old Westbury, NY, Inge-Marie Eigsti, University of Connecticut, Storrs, CT, Tina Goldsmith, Trumpet Behavioral Health, Lakewood, CO, Christine Syriopoulou, University of Macedonia, Thessaloniki, Greece, Sara Jane Webb, University of Washington, Seattle, WA.

We also want to acknowledge the "Top 10" reviewers (i.e., those individuals who provided the greatest number of comprehensive, thorough and timely reviews) for the Journal this year:
1. Kenneth Aitken
2. Brian Reichow
3. Ernst VanBergeijk
4. Johnny Matson
5. Deborah Fein
6. Lynn Koegel
7. Susan White
8. Marc Woodbury Smith
9. Christine Syriopoulou
10. David Simmons

We list below the names of all individuals who have contributed one or more reviews to the Journal this year. Although far from perfect, peer review is the most effective way we have, as a field, to ensure the quality of our 
scholarly product. We thank all these individuals for their hard work and dedication.

Fred R. Volkmar, M.D.

Editor

Abha Gupta

Adam Naples

Adelaide Robb

Agnès Lacroix

Agnies Van Eeghen

Ahmad Gharaibeh

Ailsa Russell

A. J. Schwichtenberg

Aja Murray

Ajmal Mian

Akio Wakabayashi

Alan Brown

Alan Lincoln

Alastair Clarkson

Albert Cotugno

Albert Donnay

Alberto Migliore

Alexander Kolevzon

Alexander Westphal

Alfred Chavira

Ali Sheikhani

Alice Carter

Alina Lartseva

Alison Betz

Alison Presmanes Hill

Alison Singer

Allison Nahmias

Alok Amraotkar

Alvi Azad

Alysa Doyle

Alyssa Alcorn

Amanda Bakian

Amanda Gulsrud

Amanda Laprime

Amanda Ludlow

Amanda Richdale

Amanda Steiner

Amaria Baghdadli

Ami Klin

Amie Duncan

Amir Hossein Memari

Amy Daniels

Amy Esler

Amy Fritsch

Amy Hansford

Amy Laurent

Amy Spriggs

Anat Zaidman-Zait
Andrea Witwer

Andrea Roberts

Andrea Samson

Andrei Miu

Andrew Stanfield

Andrew Whitehouse

Andy Shih

Angela Reiersen

Angelica Ronald

Anibal Gutierrez

Anjana Bhat

Anke Scheeren

Ann Neumeyer

Ann Ozsivadjian

Anna Bonnel

Anna Esbensen

Anna Järvinen

Anne Cummings

Anne Snow

Anne Wheeler

Anne-Katrin Muth

Anneke Louwerse

Anneli Kylliainen

Annelies de Bildt

Annelou de Vries

Annette Estes

Annette Joosten

Annette Karmiloff-Smith

Annika Hellendoorn

Anoek Oerlemans

Antoinette Sabatino

Antonia Hamilton

Antonio Hardan

Antonio Narzisi

Armando Bertone

Ashleigh Hillier

Ashley Johnson

Ashley Robertson

Asif Zia

Athena Lickel

Atsushi Senju

Audrey Carson

Audrey Smerbeck

Audrey Thurm

Avery Voos

Azadeh Kushki

Barbara D'Entremont

Barbara Esbjørn

Bart Boets

Beate Finger

Beatrix Labadi

Ben Alderson-Day

Ben Popple 
Ben Robins

Benedetto Vitiello

Benjamin Thomas

Benjamin Yerys

Benjamin Zablotsky

Bernard Crespi

Bertram Ploog

Beth Johnson

Beth Lanning

Beth Malow

Bethany McKissick

Bibi Huskens

Bien Lai

Bill Carlezon

Blake Hansen

Blythe Corbett

Bonita (Bonnie) Klein-Tasman

Bonnie Zima

Bradley Drysdale

Brandon Keehn

Breanne Byiers

Brenda Smith-Myles

Brenna Maddox

Brent Vander Wyk

Brian Boyd

Brian Lee

Brian Moss

Brian Reichow

Brian Skotko

Brian Willoughby

Bridget Taylor

Brittany Travers

Brooke Ingersoll

Bruce Kastin

Bruce Stevenson

Bryan Harrison

Bryan Reimer

Bryna Siegl

C. Tissot

Caitlin Conner

Calum Hartley

Cameron Neece

Camilla McMahon

Canan Sola Ozgüç

Cara Cashon

Cara Damiano

Carissa Cascio

Carl Feinstein

Carla Marin

Carla Mazefsky

Carla Wood Jackson

Carlisle Runge

Carol Schall
Carol Weitzman

Carolien Rieffe

Carolyn D. Drews

Carolyn Shivers

Carrie Ballantyne

Catherine Best

Catherine Gale

Catherine Hanson

Catherine Jones

Catherine Lord

Catherine Manning

Catherine Stamoulis

Celine Saulnier

Celine Saulnier

Chata Dickson

Cheryl Dissanayake

Cheryl Klaiman

Cheryl Klaiman

Cheryl Nye

Cheryl Sandford

Cheryl Wright

Chiara Fiorentini

Chien-Yu Pan

Ching-lin Wu

Christie Karpiak

Christina Nicolaidis

Christine Ecker

Christine Falter

Christine Peters

Christine Reeve

Christine Syriopoulou

Christopher Baglio

Christopher Cowan

Christopher Gillberg

Christopher Hammond

Christopher Lemons

Christopher McDougle

Christopher Rim

Christopher Sharpley

Chrystina Anna Dolyniuk

Ciara Foody Padden

Cihat Kagan Gurkan

Claire Dumont

Claire Vallotton

Clare Harrop

Clare Harrop

Claudia Hilton

Claudio Longobardi

Cleotilde Gonzalez

Colby Chlebowski

Colleen Hayes

Connie Kasari

Connie Sung 
Connor Kerns

Cooper Woodard

Corinna Grindle

Cory Shulman

Craig Erickson

Cynthia Johnson

Cynthia Pearl

Cyrielle Derguy

D. J. Greene

Damian Santomauro

Dan Coulter

Danica Denton

Daniel Bone

Daniel Campbell

Daniel Dickstein

Daniel Fienup

Daniel Fiset

Daniel Maggin

Daniel Molina

Daniel Rossignol

Daniel Tarquinio

Daniel Yang

Darren Hedley

Darryl Eyles

David Amaral

David Berry

David Beversdorf

David Cihak

David Cohen

David Cox

David Dunn

David Feil-Seifer

David Gast

David Hong

David Huepe

David Mandell

David Noelle

David Richman

David Simmons

David Skuse

David Trembath

David Wacker

David Williams

Dean Sutherland

Deborah Bilder

Deborah Fein

Deborah Riby

Debra Cote

Debra Kamps

Declan Murphy

Deirdra Murphy

Denis Sukhodolsky

Derek Carlson
Dermot M. Bowler

Devin Casenhiser

Diana Robins

Diana Wilde

Diane Williams

Dima Amso

Ditza Zachor

Domenic Cicchetti

Donald Oswald

Donald Rojas

Donna Murray

Donnette Cox

Doreen Granpeesheh

Doris Hill

Dorit ben Shalom

Dorit Kliemann

Dorota Mącik

Dorothy Strickland

Dorothy Stubbe

Dougal Hare

Douglas Carothers

Douglas Woods

Dustin Lamport

Dustin Sarver

E. Carter

E. Sandra Byers

E. T. Barker

Ebtissam Murshid

Ebubekir Aksay

Ed Ritvo

Edwin Cook, Jr

Eileen Haebig

Eileen Simon

Eileen Simon

Einar Ingvarsson

Elaina DaLomba

Elena Grigorenko

Eli Lebowitz

Elisabeth DeVilbiss

Elise Robinson

Elizabeth Kelley

Elizabeth Kim

Elizabeth Laugeson

Elizabeth Lorah

Elizabeth Milne

Elizabeth Pellicano

Elizabeth Perkins

Elizabeth Redcay

Elizabeth Schoen

Elizabeth Sheppard

Elizabeth Smith

Ellahe Chabani

Ellen Giarelli 
Elliott Beaton

Elysa Marco

Emily Bouck

Emily Bremer

Emily Farran

Emily Kuschner

Emily Simonoff

Emma Duerden

Emma Gowen

Emma Honey

Enjey Lin

Eric Bieniek

Eric Chessen

Eric Fombonne

Eric Hazen

Eric Morrow

Eric Olofson

Eric Storch

Erica Hinckson

Ericka Wodka

Erika Nurmi

Erin Barton

Erin Barton

Erin McCurdy

Erinn Finke

Ernst VanBergeijk

Eron Friedlander

Ervin Poljac

Esubalew Bekele

Eva Baharav

Eva Billstedt

Eva Hesselmark

Eva Loth

Eva Troyb

Evald Saemundsen

Eve Spratt

Ewa Pisula

Eynat Gal

Farah El Zein

Fatimah Bahrami

Femi Oyebode

Fernanda Fernandes

Fiona Miller

Flavia Chiarotti

Florence DiGennaro Reed

Franc Donkers

France Lainé

Frances Chen

Francesca Cirulli

Francesca Foti

Francesca Happe

Francisca van Steensel

Francois Bolduc
François Michaud

Frederick Shic

Fuk Chuen Ho

G. D. Waiter

Gabriel Dichter

Gabriela Rosenblau

Gaea Moore

Gael Orsmond

Gagan Joshi

Gail McGee

Garland Jones

Gary Mesibov

Gene Blatt

Gene Fisch

George Anderson

Gerald Goldstein

Gerrit Van Schalkwyk

Giacomo Vivanti

Gillian Baird

Gillian Baird

Gillian Hanely

Gillian Hayes

Ginny Russell

Giulia Righi

Grace Gengoux

Greg Reid

Greg Wallace

Gregory Barnes

Gregory Chasson

Gulnoza Yakubova

H. Gardener

Hanna Alonim

Hanna Kovshoff

Hanna Stevens

Hannah Cholemkery

Hannah Sowden

Hans Christoph Steinhausen

Hao Chen

Hayley Leonard

Heather Henderson

Heather Nuske

Heidi Abadeh

Helen Leonard

Helen McConachie

Henny Admoni

Herbert Roeyers

Hidetoshi Takahashi

Hideya Koshino

Hilde Geurts

Hillary Devlin

Hironori Akechi

Hitoshi Hara

Hjördís Atladóttir 
Hoffmann Thomas

Hojjat Allah Haghgoo

Hsueh-ling chang

Hsu-Min Chiang

Iain McClure

Ilene Schwartz

Iliana Magiati

Iliyana Pacheva

Inge-Marie Eigsti

Ira Cohen

Iris Gordon

Isabel Clare

Isabel Smith

Isabelle Rapin

J. Mulle

J. Olivar-Parra

J. Parker

J. J. van der Meere

J. M. Valla

Jacalyn Guy

Jacqui Rodgers

Jaime Ballard

James Adams

James Bouder

James Cauraugh

James Christopher Edgar

James Holdnack

James Lee

James Luiselli

James M. Perrin

James McCracken

James McGough

James McPartland

James Phalen

James W. Tanaka

Jan van Santen

Jane Joseph

Jane Roberts

Jane Thierfeld Brown

Janet Bang

Janice Howard

Janina Neufeld

Janine Montgomery

Janine Stichter

Janis Oram Cardy

Jariya Chuthapisith

Jason Travers

Jason Wolff

Jeff Sigafoos

Jeff Skowronek

Jeffrey Karst

Jeffrey Wood

Jenni Karl
Jennifer Foss-Feig

Jennifer Ganz

Jennifer Gillis

Jennifer Ledford

Jennifer Pfeifer

Jennifer Silk

Jennifer Symon

Jennifer Mayer

Jenny Downs

Jeremy Jewell

Jeremy Veenstra-Vanderweele

Jeroen Dewinter

Jessica Abrams

Jessica Bradshaw

Jessica Dykstra

Jessica Hellings

Jessica Hobson

Jessica Klusek

Jessica Lester

Jessica Waxler

Jewel Crasta

Jill Boucher

Jill De Villiers

Jill Fodstad

Jill Harris

Jill Hollway

Jill Locke

Jill Silverman

Jillian Greczek

Jillian Sullivan

Joana Almeida

Jocelyn LeBlanc

Jodene Fine

Joel Bregman

Joel Wu

Johan Wagemans

Johannes Rojahn

John bankart

John Constantino

John Herrington

John McGrew

John W. Harrington

Johnna Swartz

Johnny Matson

Jois Stansfield

Jon Brock

Jonathan Campbell

Jonathan Delafield-Butt

Jonathan Tarbox

Jonathan Weiss

Jonna Bobzien

Jos Hendriksen

Joseph Maes 
Joseph P. McCleery

Joseph Lucyshyn

Joshua Diehl

Joy Hirsch

Joy Xin

Joyce Suh

Judah Koller

Judith Danovitch

Judith Meyers

Judith Pijnacker

Judy Reaven

Juhi Kaboski

Juhi Pandey

Julia O'Rourke

Julie Rubin

Julie Taylor

Julie Wolf

Julie-Ann Jordan

Justin Hampton

Justin Leaf

Justin Rowberry

K. D. Veena

Kahsi Smith

Kami Koldewyn

Kang Lee

Kara Hume

Kara Styck

Karen Ashwood

Karen Bearss

Karen Dobkins

Karine Silva

Karla McGregor

Karyn Bailey

Kat Houghton

Katarzyna Chawarska

Kate Fiske

Kate Hoffman

Kate Sofronoff

Katerina Papanikolaou

Katharine Zuckerman

Katherine Bellone

Katherine Gotham

Katherine Walton

Kathleen A. Quill

Kathleen Feeley

Kathleen Hopf

Kathleen Zanolli

Kathy Koenig

Kathy Lawton

Katie Maras

Keely Cheslack-Postava

Keise Izuma

Keith Radley
Keith Shafritz

Kelly Blankenship

Kelly Whalon

Ken Gobbo

Kenneth Aitken

Kenneth Gadow

Kenneth Kobak

Kenneth Poon

Kerri Staples

Kevin Callahan

Khaled Saad

Kimberly Kroeger

Kimberly Maich

Kirsten Schohl

Klaus Libertus

Kojo Mintah

Kosuke Asada

Kristen Gillespie-Lynch

Kristen Linton

Kristen Bottema-Beutel

Kristine De Lopez

Kuan-Lin Chen

Kwang-Sun Blair

Kyle Bennett

Kyle Williams

Kylie Gray

Kyongboon Kwon

Kyung Mee Chung

L. Jarrett Barnhill

L. Eugene Arnold

Laci Watkins

Larah van der Meer

Larry Lee

Laszlo Erdodi

Latha Soorya

Laudan Jahromi

Laura Boccanfuso

Laura Crane

Laura Elliffe-Pawlowski

Laura Gutermuth Anthony

Laura O. Smith

Laura Politte

Laura Schrreibman

Laura Silverman

Lauren Adamson

Lauren Berkovits

Lauren Bishop-Fitzpatrick

Lauren Goodwin

Lauren Herlihy

Lauren Kenworthy

Lauren Miller

Lauren Richmond

Lauren Taylor 


Lauren Weiss
Lauren Little
Laurent Mottron
Laurie Sperry
Laurie Vismara
Lawrence Fung
Lawrence Scahill
Leah Stein
Leann Smith
Lee Marcus
Leif Ekblad
Leigh Chapman
Leonardo Emberti Gialloreti
Leonhard Schilbach
Leslie Carver
Leslie Rescorla
Letitia Naigles
Leyla Akoury Dirani
Li Yi
Li-Ching Lee
Lidia Gabis
Liisa Hantsoo
Linda Bambara
Linda Bandini
Linda Caterino
Linda Haymes-Elliott
Linda Heitzman-Powell
Linda LeBlanc
Linda Murdock
Linda O’Neill
Linda Pring
Linda Quirmbach
Linda Quirmbach
Linda Watson
Lindee Morgan
Lindsay Oberman
Lindsey Sterling
Li-Nien Chien
Lisa Christensen
Lisa Henderson
Lisa Honigfeld
Lisa Nowinski
Lisa Wiggins
Lizbeth Escobedo
Loes Koelewijn
Logan Hart
Logan Wink
Lolanda Leite
Longchuan Li
Lori-Ann Sacrey
Louis Timmerman Hagopian
Louise Kaczmarek

Lauren Weiss

Lauren Little

Laurent Mottron

Laurie Sperry

Laurie Vismara

Lawrence Fung

Lawrence Scahill

Leah Stein

Leann Smith

Lee Marcus

Leif Ekblad

Leigh Chapman

Leonardo Emberti Gialloreti

Leonhard Schilbach

Leslie Carver

Leslie Rescorla

Letitia Naigles

Leyla Akoury Dirani

Li Yi

Lidia Gabis

Liisa Hantsoo

Linda Bambara

Linda Bandin

Linda Caterino

es-Elliott

Linda LeBlanc

Linda Murdock

da O'Neill

Linda Quirmbach

Linda Quirmbach

Linda Watson

Lindee Morgan

Lindsay Oberman

Lindsey Sterling

Li-Nien Chien

Lisa Christensen

Lisa Henderson

Lisa Honigfeld

Lisa Nowinski

Lisa Wiggins

Lizbeth Escobedo

Loes Koelewijn

Logan Hart

Logan Wink

Lolanda Leite

Longchuan Li

Lori-Ann Sacrey

Lorna Timmerman

Louise Kaczmarek
Luc Lecavalier

Luke Tsai

Lynn Koegel

Lynn Wsterhouse

M. Van der Stel

M. Rus-Calafell

M. C. DeSoto

M. C. Lai

Madeleine Grigg-Damberger

Madeline Harms

Maithilee Kunda

Mandy Fulton

Manzar Ashtari

Mara Parellada

Marc Woodbury-Smith

Marc Woodbury-Smith

Marco Garzitto

Mareike Altgassen

Maretha De Jonge

Maretha De Jonge

Margaret Creedon

Margaret Flores

Margaret Maglione

Margo Holm

Marguerite O'Haire

Maria Casagrande

Maria Efstratopoulou

Maria Martzoukou

Maria Pia Bucci

Marianne Barton

Marianne Latinus

Marie Copploa

Marie-Christine Potvin

Marie-Ève Garon

Marie-José Dealberto

Marieke de Vries

Marielle Stel

Marientina Gotsis

Marilyn Hays

Marine Grandgeorge

Marisa Filipe

Marissa Diener

Marja-Leena Mattila

Marjorie Charlop

Marjorie Solomon

Mark Brosnan

Mark Cicero

Mark Dadds

Mark Strauss

Mark Sundberg

Mark Tommerdahl

Mark Vida

Martin Volker 
Mary E. Van Bourgondien

Mary Hediger

Masafumi Harada

Matthew Albrecht

Matthew Belmonte

Matthew Bennett

Matthew Brock

Matthew Goodwin

Matthew Hollocks

Matthew Hudson

Matthew Lerner

Matthew Maenner

Matthew Mosconi

Matthew Siegel

Matthew Smith

Matthew Walenski

Matthew Williams

Maureen Durkin

Maurice Feldman

Mayada Elsabbagh

Maysa Akbar

Meagan Talbott

Megan Boyle

Megan Freeth

Megan MacDonald

Megan Martins

Megan Tudor

Megan Farley

Meghan Burke

Meghan Miller

Meghann Lloyd

Mehmet Yanardağ

Mei-Chun Cheung

Melanie Ring

Mélina Rivard

Melissa King

Mette Elmose Andersen

Micah Mazurek

Michael Alessandri

Michael Aman

Michael D. Powers

Michael Ganz

Michael Himle

Michael John Carley

Michael Murias

Michael Bloch

Michael Cuccaro

Michael Halassa

Michael Morrier

Michael Rutter

Michael Siller

Michael Fitzgerald

Michael Leon
Michal Hrdlicka

Micheal Sandbank

Michele Bishop

Michelle Alexander

Michelle Dawson

Michelle Kandalaft

Michelle Palumbo

Michelle Patriquin

Michelle Suarez

Mickey Keenan

Miiamaaria Kujala

Mikhail Kissine

Mikle South

Mina Park

Ming Wai Wan

Miriam Boesch

Misa Kayama

Missy Teatero

Mitchell Byrne

Mohamed Abdelkader Bencherif

Mohammad Ghaziuddin

Mohammad Khayatzadeh

Molly Crossman

Molly Helt

Molly Losh

Mona Holmqvist Olander

Monica Biscaldi

Muhammad Azeem

Murray Maybery

Myra Beth Bundy

N. Russo-Ponsaran

N. Sell

Nalin Payakachat

Nancy Cheak-Zamora

Nancy Garon

Nancy Getchell

Nancy Minshew

Nancy Moss-Rascusin

Nancy Raitano Lee

Nanda Rommelse

Nanda Rommelse

Naomi Ekas

Natália Correia

Natalie Russo

Natalie Webb

Nathaniel Laor

Nava Silton

Nicholas Chown

Nicholas Falk

Nicholas Gelbar

Nicholas Riches

Nick Fieschko

Nicole Dueker 
Nicole Papadopoulos

Nicole Zurcher

Nidal Daou

Nigel Newbutt

Nirit Bauminger-Zviely

Noah Sasson

Norah Johnson

Norsiah Fauzan

Nouchine Hadjikhani

O. Megnin

Olga Solomon

Olivia Veatch

Opal Ousley

Oren Shtayermman

Ouriel Grynszpan

Paige Weinger

Pam DiLavore

Pamela Heaton

Pamela Crooke

Pamela Pallett

Pamela Ventola

Panagiotis Siaperas

Paola Rocca

Pat Mirenda

Patricia García-Primo

Patricia Howlin

Patricia Jarvis

Patricia Oliveira Lima Muñoz

Patricia Prelock

Patricia Rao

Patricia van Wijngaarden-Cremers

Patrick Craanen

Paul Cavanagh

Paul Probst

Paul R. Benson

Paul Ruvolo

Paul Wang

Paul Wehman

Paula Goldenberg

PeiFang Wu

Penny Corkcum

Per Andersen

Peter Deschamps

Peter Doehring

Peter Enticott

Peter Hobson

Peter Marschik

Peter Mitchell

Peter Mundy

Peter Tanguay

Peter W. Dowrick

Peter Yellowlees

Phil Reed
Philip Kendall

Priscilla Dseagu

Quan Wang

Quentin Guillon

R. Canitano

R. Chudal

R. Matthew Reese

R. A. Mason

R. M. Bendixen

Raanan Raz

Rachael Bedford

Rachel Jellett

Rachel Loftin

Rachel Loftin

Radha Macculloch

Rahul Bansal

Rajani Ramachandran

Rajesh Kana

Rama Novogrodsky

Ramesh Kaipa

Randi Hagerman

Randolph Blake

Raymond Romanczyk

Rebecca Jones

Rebecca Landa

Rebecca Lucas

Rebecca Schmidt

Renee Lajiness-O'Neill

Rhea Paul

Rhiannon Luyster

Rita Eagle

Riva Ritvo

Robert Drake

Robert Hendren

Robert Hodapp

Robert Horner

Robert Hurley

Robert Isenhower

Robert Koegel

Robert LaRue

Robert Pennington

Robert Rada

Robert Rieske

Robert Schultz

Roberto Tuchman

Robin Hansen

Roger Jou

Roma Vasa

Ron Thibert

Ronald Leaf

Rondalyn Whitney

Rose Mason

Roseann Schaaf 
Rosemary Waring

Roy Grinker

Rutger van der Gaag

Ruth Grossman

Ruth Hurst

Ruth Lucas

Ryan Kellems

Ryan Stevenson

Sabine Roza

Safa Zaki

Saime Tek

Sally Clifford

Sally J. Rogers

Sally Ozonoff

Samantha Landino

Samira Soliman

Samuel Odom

Samuel Sennott

Samy Azer

Sana Abu-Dahab

Sander Begeer

Sandra Harris

Sandra Hodgetts

Sandra Maestro

Sandra Maria Herondina Coelho Aguiar

Sandra Tsang

Sandra Ward

Sanja Banjavcic

Sara Spencer

Sara Webb

Sarabeth Broder-Fingert

Sarah Carlon

Sarah Carrington

Sarah Elsea

Sarah Haigh

Sarah Hurwitz

Sarah Parsons

Sarah Paterson

Sarah Schipul

Sarah Schoen

Sarah Wigham

Sarika Peters

Sarit Guttmann-Steinmetz

Sasha Walters

Sayyed Ali Samadi

Scott S. Hall

Sebastian Gaigg

Selda Ozdemir

Selin Aktan Guloksuz

Sema Batu

Seray Olcay Gul

Seung-Hyun Son

Shannon Delaney
Shannon Wagner

Sharon McLaughlin

Sheila Christopher

Sherrilene Classen

Shirli Wener

Shivani Sharma

Shota Uono

Shota Uono

Shulamite Green

Sigal Eilat-Adar

Sigan Hartley

Siglia Camargo

Sigmund Eldevik

Signe Naftel

Silvio Loddo

Simon Baron Cohen

Siobhan Barry

Skyler Hawk

Sloane Burgess

So Hyun Kim

Soile Loukusa

Somer Bishop

Sonia Martínez-Sanchis

Stacey Reynolds

Stacy Shumway

Stefanie Bodison

Stefano Taddei

Stelios Georgiades

Stephanie Ameis

Stephanie Bader

Stephanie Bryson

Stephanie Hayes

Stephanie Malone

Stephanie Sansone

Stephanie Shire

Stephen Buka

Stephen Deutsch

Stephen Edward McMillin

Stephen Kanne

Stephen Oller

Steve Kroupa

Steven Gutstein

Stewart Mostofsky

Sue Cobb

Sue Fletcher-Watson

Susan Assouline

Susan Calhoun

Susan Campbell

Susan Carter

Susan Connors

Susan Ellis Weismer

Susan Faja

Susan Hyman 
Susan Jarmuz-Smith

Susan Leekam

Susan Levy

Susan Milne

Susan Peppe

Susan Perlman

Susan Vig

Susan White

Susanna Mierau

Susanna Villa

Susanne Bejerot

Suzanna Russell-Smith

Suzannah Iadarola

Suzanne Macari

Sven Bolte

Sven Sandin

Sydney Pettygrove

Sylvia Kwok

Sylvie Beauchamp

Sylvie Goldman

Takeshi Nishiyama

Tamara Germani

Tanja Sappok

Tanushree Saxena

Tatiana Assumpção

Taylor Day

Teal Benevides

Ted Hutman

Telma Almeida

Teresa Bennett

Teresa Burrell

Teresa Tavassoli

Teresa Tavassoli

Teri Belkin

Terje Falck-Ytter

Terry Au

Terry Gibbs

Terry Katz

Terry Overton

Terry Overton

Tess Clifford

Thayne Sweeten

Thierry Chaminade

Thomas Dratsch

Thomas L. Layton

Thomas Ollendick

Thomas Anders

Thomas Berney

Thomas Fabricius

Thomas Frazier

Thusha Rajendran

Tiffany Hutchins

Tiffany Rogers
Timothy Hartshorne

Timothy Yu

Tina Dyches

Tina Goldsmith

Tjeerd Jellema

Todd Levine

Tony Charman

Tonya Davis

Tove Lugnegard

Trine Lise Bakken

Tristram Smith

Ty Vernon

Tychele Turner

Tze Jui Goh

Uttama Lahiri

V. Demicheli

Valerie Gaus

Valerie Shafer

Valérie Van Hees

Valsamma Eapen

Vanessa Green

Vanessa Hus

Vanessa Troiani

Vasiliki Totsika

Vicki Bitsika

Vickie Pasterski

Victoria Shea

Victoria Hallett

Victoria Knight

Victoria Talwar

Viktoria Lyons

Virginia Chaidez

Virginia Wong

Virginie Cobigo

Vlatka Boričević Maršanić

Walter Kaufmann

Walton Roth

Wataru Sato

Wendy Blumenthal

Wendy Silverman

Wendy Machalicek

Whitney Relf

William Mandy

William Hudenko

William Lohr

William Sharp

Winnie Yu Pow Lau

Winnie Dunn

Wouter De la Marche

Xin Tong

Xin Wei

Ya-Chih Chang

Yael Kimhi 
Yamini Howe

Yanki Yazgan

Yatin Mahajan

Yawei Cheng

Yeaton Clifton
Yoed Kenett

Yogeswara Modugumudi

Yoko Kamio

Zachary Warren

Zsuzsa Kaldy 